\title{
Frontal Sinus as an aid in Gender Identification in Forensic Dentistry: A Retrospective Study using Cone Beam Computed Tomography
}

\author{
${ }^{1}$ Ceena Denny, ${ }^{2}$ Anu S Jacob, ${ }^{3}$ Junaid Ahmed, ${ }^{4}$ Srikant Natarajan, ${ }^{5}$ Almas Binnal, ${ }^{6}$ Nanditha Sujir
}

\section{ABSTRACT}

Aim: The aim of our study was to measure the frontal sinus morphology that could aid us in gender determination and also to assess the difference in measurements between the right and left frontal sinus.

Materials and methods: A retrospective study was done using 100 cone beam computed tomography (CBCT) images ( 50 males and 50 females) matched with age and gender with full field of view (FOV). The examinations were carried out using Promax 3DMid (Planmeca Oy., Helsinki, Finland) CBCT unit. The frontal sinus was assessed in coronal, sagittal, and axial planes, and the maximum measurements in each section were recorded. The results to compare the right and left frontal sinus were analyzed using paired t-tests, and independent Student's t-test was used to compare the difference in measurements of frontal sinus between males and females.

Results: We found that the left side of the frontal sinus was bigger than the right side, and while comparing between the genders, it was found that the measurements were greater in males. Statistically significant results were obtained on comparing between the sides and gender.

Conclusion: As mentioned in previous studies, frontal sinus measurements are significantly higher in males compared with females which can, therefore, be used in gender identification in cases of mass disasters.

Clinical significance: Frontal sinus measurements can be used as an adjunct in gender identification in mass disasters and with advances in technology. Cone beam computed tomography, in addition to providing accurate measurements, has overcome all the disadvantages with two-dimensional imaging.

Keywords:Cone beam computed tomography, Forensic investigations, Frontal sinus, Gender determination.

How to cite this article: Denny C, Jacob AS, Ahmed J, Natarajan S, Binnal A, Sujir N. Frontal Sinus as an aid in Gender Identification in Forensic Dentistry: A Retrospective Study using Cone Beam Computed Tomography. World J Dent 2018;9(1):34-37.

\footnotetext{
1-3,5,6 Department Oral Medicine and Radiology, Manipal College of Dental Sciences, Manipal Academy of Higher Education Mangaluru, Karnataka, India

${ }^{4}$ Department of Oral and Maxillofacial Pathology, Manipal College of Dental Sciences, Manipal Academy of Higher Education, Mangaluru, Karnataka, India

Corresponding Author: Anu S Jacob, Department Oral Medicine and Radiology, Manipal College of Dental Sciences Manipal Academy of Higher Education, Mangaluru, Karnataka India, e-mail: anusarajacob@ymail.com
}

Source of support: Nil

Conflict of interest: None

\section{INTRODUCTION}

Cone beam computed tomography as an imaging tool in dentistry has varied clinical applications, one of which is viewing sinuses. The accuracy of measurements using CBCT is also commendable. The basic two-dimensional imaging though vastly used has disadvantages like superimpositions and changes in the quality of image secondary to positioning errors and processing the films.

The frontal sinus shows no change after the age of 20 and remains stable throughout the individual's life until old age, when gradual pneumatization can occur from atrophic changes. ${ }^{1}$ Similar to fingerprints, the frontal sinus is very unique in every individual, even monozygotic twins. Therefore, frontal sinus radiographs can be compared to make the identification a simple, swift, and an unambiguous procedure. ${ }^{2}$ Camargo et $\mathrm{al}^{3}$ in their article stated that correlation of the morphology of the frontal sinus with gender shows that the frontal sinus is smaller in women, an aspect that points out its unique characteristic and importance in human identification. Imaging of the human body, especially the head and neck region, has been useful in forensic dentistry for proving medicolegal cases and inhuman identifications during mass disasters. Hence, this study was done to measure the frontal sinus morphology, which in turn helps us in gender determination.

\section{MATERIALS AND METHODS}

A retrospective study was conducted after obtaining clearance from the Institutional Ethics Committee. One hundred CBCT images (50 males and 50 females) matched with age and gender with full FOV were retrieved. Information regarding age, sex, and systemic conditions, etc., of the study subjects was collected from their dental records. The subjects included in the study were healthy individuals without any pathology in the sinus and patients in the age range of 20 to 30 years. The reason for age criteria was due to the fact that the growth of frontal sinus completes or attains its development by 20 years and as age progresses the walls become thinner and hence, appears larger. The patients excluded from the study were those 
who reported with history of trauma or pathology to the sinus, absence of sinus unilaterally or bilaterally, syndrome patients, patients with congenital disorders and patients with history of surgical intervention, such as orthognathic surgery, sinus lift, or any surgery. The examinations were carried out using the Promax 3DMid (Planmeca Oy., Helsinki, Finland) CBCT unit. The radiographs were taken at $90 \mathrm{kVp}, 8 \mathrm{~mA}$, and an exposure time of 27 seconds. A slice thickness of $0.400 \mathrm{~mm}$ was used to assess the sections. The exposure parameters according to the standard default values were based on the FOV.

The frontal sinus was assessed in coronal, sagittal, and axial planes, and the maximum measurements in each section were recorded. The height and width of the frontal sinus were measured. The coronal sections were assessed for maximum superoinferior and mesiodistal measurements for both right and left frontal sinus (Fig. 1). The measurements for right and left frontal sinus in the sagittal section, i.e., maximum superoinferior measurement, were established from the patient's medial line and the measurements taken were correlated with the coronal sections. The maximum anteroposterior (AP) measurements were recorded for right and left frontal sinus after correlation with the axial section. In the axial section, the AP measurement was recorded after correlating it with the sagittal section, and mesiodistal measurements were taken at a section where maximum mesiodistal measurements were available.

The results to compare the right and left frontal sinus were analyzed using paired t-tests, and independent Student's t-test was used to compare the difference in measurements of frontal sinus between males and females.

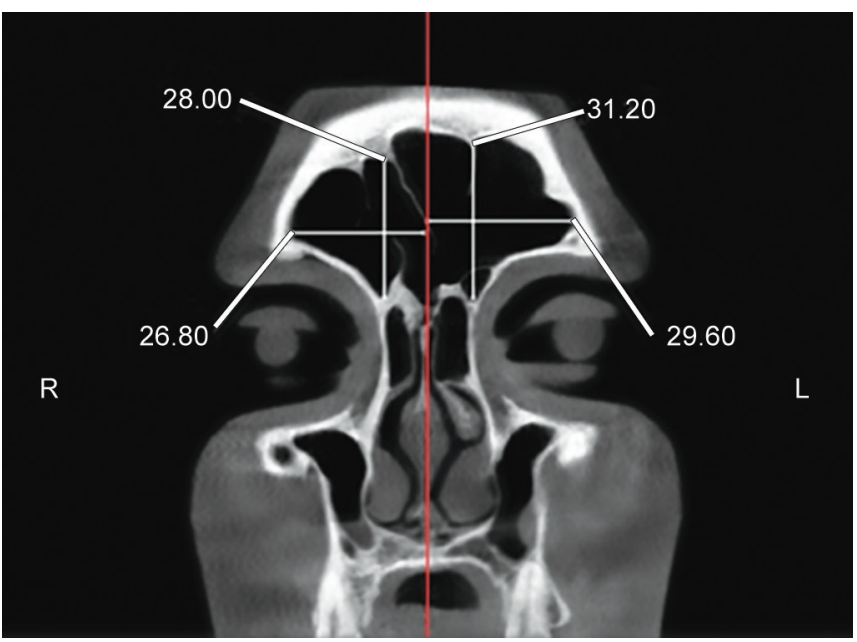

Fig. 1: Frontal sinus measurement superoinferiorly and mesiodistally in coronal section

\section{RESULTS}

\section{Paired t-test}

The paired t-test was applied to compare the difference in measurements of right and left frontal sinus in axial, coronal, and sagittal sections irrespective of gender. One hundred patients were included in the study, which was divided into 50 males and 50 females (Table 1).

\section{Axial Section}

On comparison of the mean values in the axial section anteroposteriorly on both right and left side, it was seen that the mean values in the axial section of left side were higher with a mean difference of 0.9698 , which was

Table 1: Total group irrespective of gender comparison of the right and left frontal sinus: paired t-test

\begin{tabular}{|c|c|c|c|c|c|c|c|}
\hline & Mean & $n$ & $\begin{array}{l}\text { Standard } \\
\text { deviation }\end{array}$ & $\frac{\text { Paired differences }}{\text { Mean difference } \pm S D}$ & $t$-value & $d f$ & $p$-value \\
\hline \multicolumn{8}{|l|}{ Pair 1} \\
\hline Axial section right AP & 9.8822 & 100 & 4.823391 & $-0.9698 \pm 3.352842$ & -2.892 & 99 & 0.005 \\
\hline Axial section left AP & 10.852 & 100 & 5.120162 & & & & \\
\hline \multicolumn{8}{|l|}{ Pair 2} \\
\hline Axial section right MD & 22.2003 & 100 & 8.410006 & $-0.286 \pm 18.737443$ & -0.327 & 99 & 0.744 \\
\hline Axial section left MD & 22.4864 & 100 & 7.542099 & & & & \\
\hline \multicolumn{8}{|l|}{ Pair 3} \\
\hline Sagittal section right AP & 11.4504 & 100 & 5.703515 & $-0.824 \pm 3.512907$ & -2.346 & 99 & 0.021 \\
\hline Sagittal section left AP & 12.2744 & 100 & 5.279986 & & & & \\
\hline \multicolumn{8}{|l|}{ Pair 4} \\
\hline Sagittal section right SI & 24.3642 & 100 & 9.612597 & $-1.6842 \pm 5.668888$ & -2.971 & 99 & 0.004 \\
\hline Sagittal section left SI & 26.0484 & 100 & 9.336425 & & & & \\
\hline \multicolumn{8}{|l|}{ Pair 5} \\
\hline Coronal section right SI & 23.8624 & 100 & 8.419029 & $-2.0716 \pm 10.3888$ & -1.994 & 99 & 0.049 \\
\hline Coronal section left SI & 25.934 & 100 & 11.02097 & & & & \\
\hline \multicolumn{8}{|l|}{ Pair 6} \\
\hline Coronal section right MD & 21.944 & 100 & 7.627122 & $-0.454 \pm 7.990745$ & -0.568 & 99 & 0.571 \\
\hline Coronal section left MD & 22.398 & 100 & 7.545016 & & & & \\
\hline
\end{tabular}

SD: Standard deviation; SI: Superoinferiorly, MD; Mesiodistally; df: Degree of freedom 
statistically significant ( $p=0.005)$. While comparing the mean values of right and left side mesiodistally, the mean value on the left side mesiodistally was higher with a mean difference of 0.2861 , which was statistically insignificant.

\section{Sagittal Section}

In this section on comparing both sides anteroposteriorly and superoinferiorly, it was found that the mean value on the left side was higher with a mean difference of 0.824 anteroposteriorly, which was statistically significant with a p-values $=0.021$ and 1.6824 superoinferiorly with significant $\mathrm{p}=0.004$.

\section{Coronal Section}

On comparison of the mean values of right and left side superoinferiorly, it was found that the mean value on the left side was higher with a mean difference of 2.0716, which was statistically significant $(p=0.049)$. It was found that the mean value on the left side was higher mesiodistally, with a mean difference of 0.454 , which showed statistically significant $\mathrm{p}=0.571$.

\section{Independent Student's t-test}

This test was done to compare the difference in measurements of frontal sinus between males and females (Table 2).

\section{Axial Section}

Comparison of the right and left frontal sinus measurements anteroposteriorly and mesiodistally between the two groups showed that both the measurements were greater in the males. The $\mathrm{p}$ values for right $\mathrm{AP}$, mesiodistal and left AP measurements are $<0.001$ and the left mesiodistal measurement had $p=0.007$, all of which were statistically significant.

\section{Sagittal Section}

Comparison of the right and left frontal sinus measurements anteroposteriorly and superoinferiorly between the two groups showed that both the measurements were greater in the males. The $p$ values for right and left AP measurements were $<0.001$ and the right and left superoinferior measurements had a $\mathrm{p}=0.141$ and 0.082 respectively, which was statistically nonsignificant.

\section{Coronal Section}

Comparison of the right and left frontal sinus measurements superoinferiorly and mesiodistally between the two groups showed that both the measurements were greater in the males. The $\mathrm{p}$ values for right and left mesiodistal measurements were $<0.001$, and the right and left superoinferior measurements had a $\mathrm{p}=0.243$ and 0.805 respectively, which was statistically nonsignificant.

Table 2: Independent t-test for comparison of frontal sinus among both the genders

\begin{tabular}{|c|c|c|c|c|c|c|}
\hline & Sex & $n$ & Mean $\pm S D$ & $t$-value & $d f$ & $p$-value \\
\hline \multirow[t]{2}{*}{ Axial section right $A P$} & $\mathrm{M}$ & 50 & $12.252 \pm 4.826764$ & 5.621 & 98 & $<0.001$ \\
\hline & $\mathrm{F}$ & 50 & $7.5124 \pm 3.49946$ & & & \\
\hline \multirow[t]{2}{*}{ Axial section right MD } & M & 50 & $25.12 \pm 6.749513$ & 3.686 & 98 & $<0.001$ \\
\hline & $\mathrm{F}$ & 50 & $19.2806 \pm 8.941291$ & & & \\
\hline \multirow[t]{2}{*}{ Axial section left AP } & M & 50 & $13.156 \pm 5.828717$ & 5.02 & 71.253 & $<0.001$ \\
\hline & $\mathrm{F}$ & 50 & $8.548 \pm 2.856517$ & & & \\
\hline \multirow[t]{2}{*}{ Axial section left MD } & M & 50 & $24.4924 \pm 7.491728$ & 2.746 & 98 & 0.007 \\
\hline & $\mathrm{F}$ & 50 & $20.4804 \pm 7.1126$ & & & \\
\hline \multirow[t]{2}{*}{ Sagittal section right AP } & M & 50 & $13.7916 \pm 5.974485$ & 4.483 & 98 & $<0.001$ \\
\hline & $\mathrm{F}$ & 50 & $9.1092 \pm 4.3409$ & & & \\
\hline \multirow[t]{2}{*}{ Sagittal section right SI } & M & 50 & $25.7844 \pm 7.681863$ & 1.486 & 87.111 & 0.141 \\
\hline & $\mathrm{F}$ & 50 & $22.944 \pm 11.11587$ & & & \\
\hline \multirow[t]{2}{*}{ Sagittal section left AP } & M & 50 & $14.5044 \pm 6.210793$ & 4.641 & 67.593 & $<0.001$ \\
\hline & $\mathrm{F}$ & 50 & $10.0444 \pm 2.757305$ & & & \\
\hline \multirow[t]{2}{*}{ Sagittal section left SI } & M & 50 & $27.6724 \pm 8.743145$ & 1.758 & 98 & 0.082 \\
\hline & $\mathrm{F}$ & 50 & $24.4244 \pm 9.710389$ & & & \\
\hline \multirow[t]{2}{*}{ Coronal section right SI } & M & 50 & $24.852 \pm 5.580843$ & 1.178 & 74.676 & 0.243 \\
\hline & $\mathrm{F}$ & 50 & $22.8728 \pm 10.49105$ & & & \\
\hline \multirow[t]{2}{*}{ Coronal section right MD } & M & 50 & $25.172 \pm 5.816095$ & 4.653 & 98 & $<0.001$ \\
\hline & $\mathrm{F}$ & 50 & $18.716 \pm 7.90196$ & & & \\
\hline \multirow[t]{2}{*}{ Coronal section left SI } & M & 50 & $26.208 \pm 8.524247$ & 0.247 & 98 & 0.805 \\
\hline & $\mathrm{F}$ & 50 & $25.66 \pm 13.13722$ & & & \\
\hline \multirow[t]{2}{*}{ Coronal section left MD } & M & 50 & $24.824 \pm 7.348318$ & 3.38 & 98 & 0.001 \\
\hline & $\mathrm{F}$ & 50 & $19.972 \pm 7.000526$ & & & \\
\hline
\end{tabular}

SD: Standard deviation, SI: Superoinferiorly, MD: Mesiodistally; df: Degree of freedom 


\section{DISCUSSION}

Frontal sinus is a hollow cavity in the frontal bone, and it is unique to each person. Like fingerprints, frontal sinus is unique in each person, even in monozygotic/dizygotic twins. Compared with fingerprints, frontal sinus assessment is preferred as soft tissues can get destroyed or mutilated when it gets burnt, ${ }^{4}$ and its arch-shaped nature with internal bony structure protects it from getting decomposed or damaged even in high impact accidents. Frontal sinuses also remain stable throughout human life. Hence, it is preferred in forensic investigations. ${ }^{5}$

In this study, on comparing the left and right frontal sinuses irrespective of gender, we found that left side was larger when compared with the right side, which is in accordance to the study conducted by other studies by Rubira-Bullen et $\mathrm{al}^{6}$ and Soman et al. ${ }^{7}$ The reason for the asymmetry in individuals of the same age could be due to the fact that both the sinuses develop independently and have different rates of osseous resorption, ${ }^{3,8-10}$ and our result was not in accordance to the study conducted by Belaldavar et $\mathrm{al}^{4}$ where they found it vice versa.

The difference in the frontal sinuses among sexes could be due to factors, such as genetic followed by muscular, nutritional, and hormonal. ${ }^{11,12}$ Our study showed that frontal sinuses in males were larger than in females, which was similar to studies conducted by Camargo et $\mathrm{al}_{1}{ }^{3}$ Belaldavar et al, ${ }^{4}$ and Rubira-Bullen et al. ${ }^{6}$

According to our study, we found that males had a larger frontal sinus and the left side of the frontal sinus was larger than the right. These findings help us in sex determination in mass disasters, crime scene, or if the body is severely decomposed. Although the radiation exposure to the patient using three dimensions is much more compared with two dimensions, the benefits outweigh the disadvantage, which makes CBCT a valuable tool in assessing the anatomy of oral and maxillofacial structures.

\section{REFERENCES}

1. Yoshino M, Miyasaka S, Sato H, Seta S. Classification system of frontal sinus patterns by radiography. Its application to identification of unknown skeletal remains. Forensic Sci Int 1987 Aug;34(4):289-299.

2. Tang JP, Hu DY, Jiang FH, Yu XJ. Assessing forensic applications of the frontal sinus in a Chinese han population. Forensic Sci Int 2009 Jan;183(1-3):104.e1-104.e3.

3. Camargo JR, Daruge E, Prado FB, Caria PH, Alves MC, Silva RF, Daruge E Jr. The frontal sinus morphology in radiographs of Brazilian subjects: its forensic importance. Braz J Morphol Sci 2007;24(4):239-243.

4. Belaldavar C, Kotrashetti VS, Hallikerimath SR, Kale AD. Assessment of frontal sinus dimensions to determine sexual dimorphism among Indian adults. J Forensic Dent Sci 2014 Jan;6(1):25-30.

5. Patil N, Karjodkar FR, Sontakke S, Sansare K, Salvi R. Uniqueness of radiographic patterns of the frontal sinus for personal identification. Imaging Sci Dent 2012 Dec;42(4):213-217.

6. Rubira-Bullen IR, Rubira CM, Sarmento VA, Azevedo RA. Frontal sinus size on facial plain radiographs. J Morphol Sci 2010;27(2):77-81.

7. Soman BA, Sujatha GP, Lingappa A. Morphometric evaluation of the frontal sinus in relation to age and gender in subjects residing in Davangere, Karnataka. J Forensic Dent Sci 2016 Jan-Apr;8(1):57.

8. Fatu C, Puisoru M, Rotaru M, Truta AM. Morphometric evaluation of the frontal sinus in relation to age. Ann Anat 2006 May;188(3):275-280.

9. Gulisano M, Pacini P, Orlandini GE. Frontal sinus dimensions in relation to the cranial index: anatomo radiologic findings. Boll Soc Ital Biol Sper 1978 Jan;54(1):66-69.

10. Nambiar P, Naidu MD, Subramaniam KA. Anatomical variability of the frontal sinuses and yours applications on forensic identification. Clin Anat 1999;12(1):16-19.

11. Quatrehomme G, Pierre F, Sapanet M, Grevin G, Paul B, Amedee O. Identification of frontal sinus pattern in forensic anthropology. Forensic Sci Int 1996 Dec;83(2):147-153.

12. Patil K, Mody RN. Determination of the sex by discriminant function analysis and stature by regression analysis: a lateral cephalometric study. Forensic Sci Int 2005 Jan;147(2-3): 125-180. 